\title{
Silent Maxillary Sinus Syndrome
}

\section{Brittney M Brady*}

Illinois College of Optometry, United States

*Corresponding Author: Brittney M Brady, Illinois College of Optometry, United States.
Received: March 19, 2021

Published: April 23, 2021

(C) All rights are reserved by Brittney $\mathbf{M}$

Brady.

\section{Abstract}

Silent Sinus Syndrome is an often-overlooked diagnosis and may serve as a diagnostic challenge for clinicians unaware of the condition. Caused by an accumulation of fluid within the maxillary sinus, formation of a negative pressure gradient leads to eventual inward displacement of the sinus walls and downward displacement of the orbital floor. Increased orbital volume, due to reduced maxillary sinus size, may result in painless enophthalmos and hypoglobus on the effected side. Patients may present with complaints of a mild ptosis, facial asymmetry, or vertical diplopia. Although a diagnosis of exclusion, SSS must be considered in the differential diagnosis of any patient presenting with such findings. Neuroimaging is considered essential to make this diagnosis.

Keywords: Silent Maxillary Sinus; SSS; Enophthalmos; Hypoglobus

\section{Abbreviations}

SSS: Silent Sinus Syndrome; ONH: Optic Nerve Head; OD: Right Eye; OS: Left Eye; OU: Both Eyes; APD: Afferent Pupillary Defect; FTMH: Full-thickness Macular Hole; CRXT: Constant Right Exotropia

\section{Introduction}

Silent sinus syndrome (SSS) is a rare condition resulting in unilateral, painless enophthalmos and hypoglobus secondary to increased orbital volume. It most often presents between the third to fifth decade of life and shows no gender predilection [1-3]. Patients are typically unaware of any previous sinus disease and present with complaints of unilateral ptosis or facial asymmetry without history of trauma [2-5]. Clinically, deepened superior lid sulcus, upper lid retraction, and hypoglobus can be observed. Few cases of vertical diplopia or restricted motility have been reported [5]. It has been theorized that occlusion of the ostiomeatal complex causes an accumulation of fluid and resultant negative pres- sure within the maxillary sinus. This force leads to a vacuum effect allowing for inward retraction of the sinus walls including the orbital floor [1-3]. The definitive diagnosis of SSS is confirmed with neuroimaging $[1,2]$.

\section{Case Report}

A 75-year-old African American male presented for evaluation of optic nerve head (ONH) pallor right eye (OD) greater than left eye (OS). He had a longstanding diagnosis of strabismic amblyopia secondary to a large angle constant right exotropia (CRXT). In addition, his ocular history was positive for bilateral pseudophakia, a retinal hole OD which had undergone unremarkable barricade laser retinopexy several years prior, and a full-thickness macular hole (FTMH) OD which had undergone repair with a pars plana vitrectomy and membrane peel in 2013. The patient's medical history was remarkable for poorly controlled HTN, pancreatitis, irregular heartbeat, acid reflux, and seasonal allergies. His systemic medications included Diltiazem Hydrochloride, gabapentin, and Prilosec. 
The patient was oriented to person, place, and time and was of normal affect. Best corrected distance vision was 20/125 OD and $20 / 50$ OS, pinhole no improvement OD or OS. Pupils were equal, round, and reactive to light with a $1+$ afferent pupillary defect (APD) OD. Confrontation visual fields, extraocular motilities, and slit lamp exam were unremarkable. Gross examination revealed mild facial asymmetry with a small ptosis OS and $2 \mathrm{~mm}$ of left hypoglobus (Figure 1). Hertel Exophthalmometry was performed, and a result was obtained of base 102 with 16 millimeters OD and 13 millimeters OS ( 3 millimeters enophthalmos OS). Manual blood pressure was measured as 154/89 millimeters of mercury on the right arm, seated. A dilated fundus exam revealed a clear vitreous, ONHs which were flat with distinct margins and diffuse pallor OD greater than OS (Figure 2). There was pigment disruption to the macula of the right eye related to the previous FTMH, macula OS was unremarkable. Vessels showed mild attenuation. The peripheral fundus was unremarkable in both eyes besides a retinal hole superior-nasal OD which was fully surrounded by barricade laser.

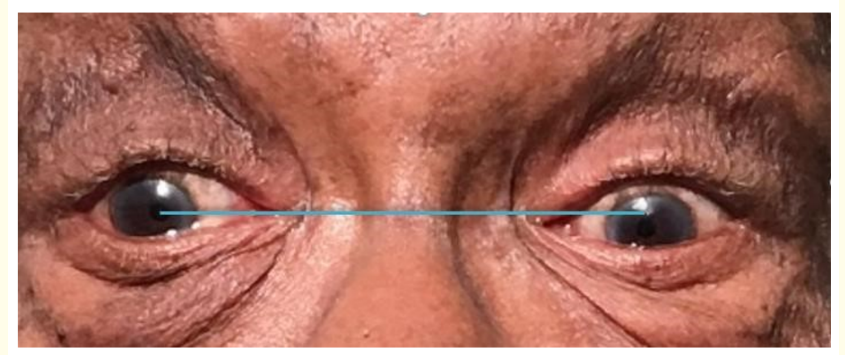

Figure 1: Left hypoglobus measuring $2 \mathrm{~mm}$ with a large angle right exotropia.

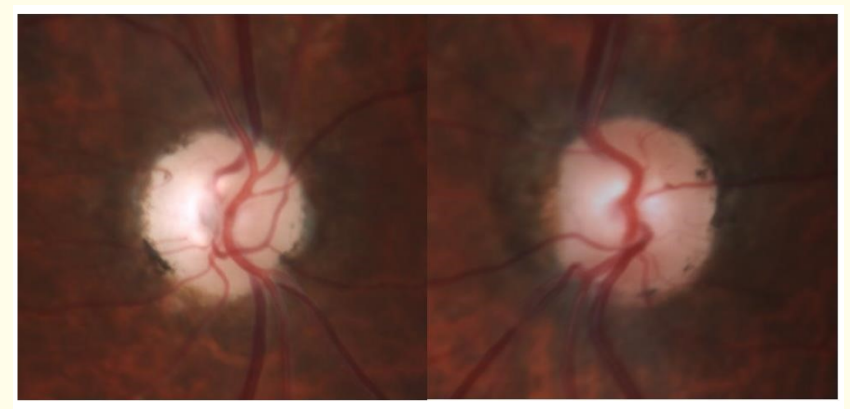

Figure 2: Right and left optic nerve heads which are flat with distinct margins and diffuse pallor greater OD and OS.
The patient denied any recent changes to vision, symptoms of diplopia, headache, malaise, jaw claudication, scalp tenderness, or concerns regarding cosmesis. With high suspicion of ischemic optic neuropathy caused by uncontrolled hypertension, the patient was scheduled for an MRI of brain and orbits both with and without contrast. Incidental exam findings of a small ptosis OS along with hypoglobus and enophthalmos OS strengthened the recommendation for an MRI. Several diagnoses were considered related to the patient's facial asymmetry, enophthalmos and hypoglobus OS. Orbital floor fracture was ruled out as there was no history of trauma or motility restrictions noted on exam. Myasthenia Gravis was ruled out as the patient denied any progressive or fluctuating symptoms particularly toward the end of the day. Horner's syndrome and cranial nerve three palsy were ruled out due to symmetric, equally reactive pupils and lack of extraocular muscle restrictions. Giant Cell arteritis was considered due to the patient's age, however no systemic symptoms were reported.

A diagnosis of ischemic optic neuropathy was confirmed with MRI findings showing sequelae from moderate chronic microangiopathic disease without signs acute disease or space occupying lesions. Additional incidental findings included: enophthalmos of the left globe, depression of the left lamina papraycea and a small left maxillary sinus with mucosal thickening and fluid accumulation. Such findings are consistent with a diagnosis of SSS and account for the mild facial asymmetry, hypoglobus OS, and enophthalmos OS.

\section{Discussion}

First described by Montgomery in 1964, the term SSS was coined by Soparkar and colleagues in 1994 [1]. Blockage of the ostiomeatal complex inhibits maxillary sinus drainage leading to an accumulation of fluid and low-grade inflammation [1-3]. This fluid buildup causes a negative pressure gradient within the sinus and resultant inward displacement of the sinus walls and downward displacement of the ipsilateral orbital floor. Spontaneous, painless enophthalmos and hypoglobus of the ipsilateral globe may develop because of the increased orbital volume [1-7]. Many patients are asymptomatic for such sinus changes as they occur gradually, hence the term "silent" in the name SSS $[2,4]$.

While the most common cause of enophthalmos is trauma in the form of a blow-out fracture [3], SSS patients with enophthalmos typically do not have a history of such trauma [2,3]. Physical examination of patients with SSS may reveal upper lid retraction, 
deepened superior lid sulcus, mild enophthalmos, and hypoglobus without any motility restrictions. Patients often present with only mild ocular symptoms or may be completely asymptomatic. Mild vertical diplopia may be reported due to hypoglobus of the effected eye. Additionally, cosmetic concerns regarding ptosis, enophthalmos, or contralateral proptosis may be reported [1-5]. The patient described in this case had no reports of diplopia likely due to the reduced vision and suppression OD as a result of a longstanding CRXT and previous FTMH. Additionally, he had no cosmetic concerns.

Neuroimaging is crucial in the diagnosis of SSS to rule out other neurological causes for the associated ocular findings [1,2]. Choosing the appropriate image modality is dependent on the goals of the test. Patients are spared from excessive ionizing radiation with MRI scans which provide exceptional detail of soft tissues. Alternatively, CT scans provide better bony detail, are faster and cheaper to perform, but expose patients to ionizing radiation [2]. Regardless of the type of imaging, typical radiological findings of SSS include ipsilateral maxillary sinus hypoplasia, opacification and inward displacement of the sinus walls, mucosal thickening, fluid accumulation, downward retraction of the orbital floor, and blockage of the ostiomeatal complex [1-5]. Such findings were reported on the MRI as incidental findings for the patient in this case (Figure 3-5). The patient was diagnosed with silent sinus syndrome based on these radiologic findings.

The main treatment for symptomatic SSS involves functional endoscopic sinus surgery [2-5]. The goals of surgery include relieving the blockage within the ostium and removal of accumulated fluid allowing for reventilating of the sinus. Typically, regaining normal sinus drainage and aeration are enough to allow the sinus walls, including the orbital floor, to return to their normal position [2]. In some cases, a secondary orbital floor reconstruction procedure may be indicated either during sinus surgery or shortly after [2-5]. As the patient described in this case was unbothered by his cosmesis and did not present with diplopia, no surgical intervention was advised. Serial exophthalmometry will be performed to monitor for progressive changes.

\section{Conclusion}

Silent Sinus Syndrome, although uncommon, must be considered in the diagnosis of a patient with unilateral ptosis, enophthal-

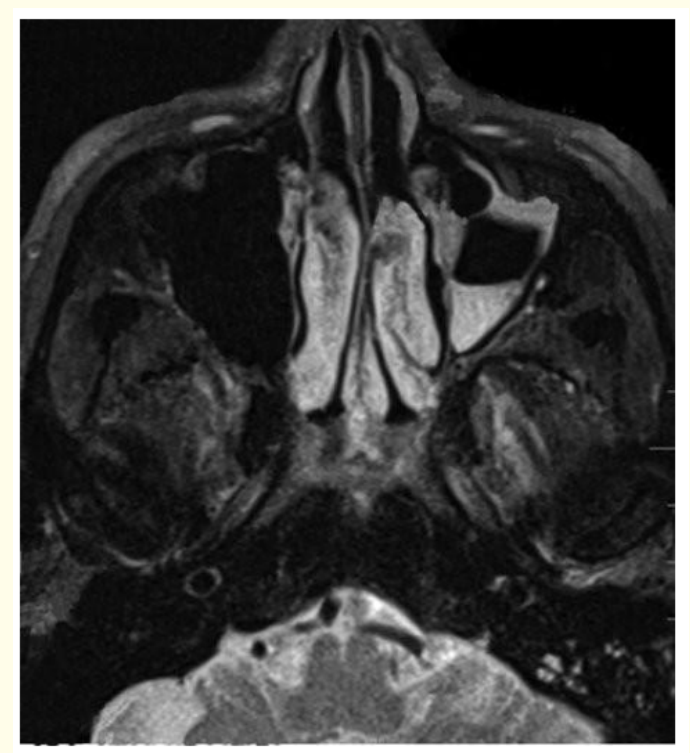

Figure 3: Axial MRI image showing a hypoplastic left maxillary sinus with thickened mucosal tissue, inward bowing of the sinus walls, and a leftward displacement of the nasal septum.

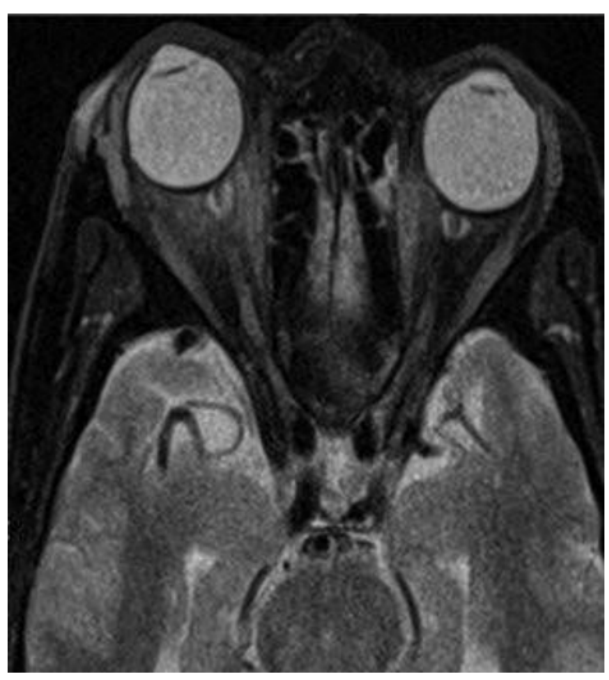

Figure 4: Axial MRI image showing enophthalmos of the left globe. 


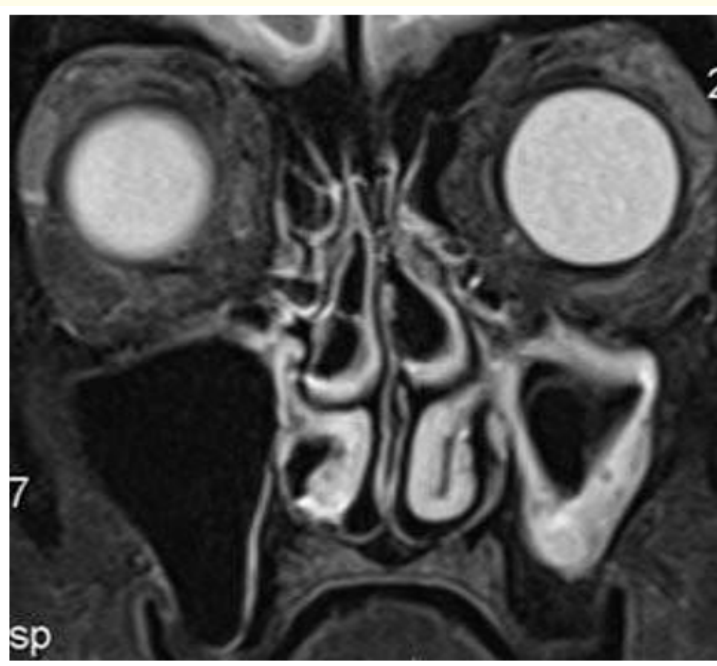

Figure 5: Coronal MRI image showing a hypoplastic left maxillary sinus with thickened mucosal tissue, inward bowing of the sinus walls, retraction of the orbital floor, and a leftward displacement of the nasal septum.

mos, hypoglobus, or vertical diplopia. This case demonstrates the classic clinical and radiologic findings of this condition. Because this patient was asymptomatic and the diagnosis of silent sinus syndrome was made based on incidental exam and radiologic findings, no treatment was recommended. However, any symptomatic patient must have diagnostic confirmation with neuroimaging prior to treatment with functional endoscopic sinus surgery.

\section{Acknowledgement}

Thank you to Dr. Leonard Messner for his assistance with the care of this patient.

\section{Bibliography}

1. Soparkar CN., et al. "The silent sinus syndrome. A cause of spontaneous enophthalmos". Ophthalmology 101.4 (1994): 772-778.

2. Mullen TV., et al. "Silent Sinus Syndrome: An Uncommon Cause of Diplopia”. Optometric Clinical Practice 2.1 (2020).

3. Gill H and Silkiss R. "Diagnosis and Management of Silent Sinus Syndrome". American Academy of Ophthalmology (2011).
4. Pula JH and Mehta M. "Silent sinus syndrome". Current Opinion in Ophthalmology 25.6 (2001): 480-484.

5. Suh JD., et al. "Bilateral silent sinus syndrome". Ear, Nose and Throat Journal: SAGE Journals 12 (2012): E19-E21.

\section{Assets from publication with us}

- Prompt Acknowledgement after receiving the article

- Thorough Double blinded peer review

- Rapid Publication

- Issue of Publication Certificate

- High visibility of your Published work

Website: $w$ ww.actascientific.com/

Submit Article: www.actascientific.com/submission.php

Email us: editor@actascientific.com

Contact us: +919182824667 